\title{
Enfoque de productividad y mejora en el ingeniero industrial de San Marcos. Estudio exploratorio para competitividad de categoría mundial
}

\section{RESUMEN}

En el propósito de resultados óptimos en su desempeño, el ingeniero industrial considera la gestión de procesos, recursos humanos y materiales buscando la productividad y eficiencia, también la gestión de oportunidades, el primero se enfoca en el sistema en equilibrio y su funcionamiento, el segundo es el desarrollo de herramientas y técnicas de mejora continua que caracteriza a la profesión. El objetivo es mostrar la propensión del ingeniero industrial hacia la estabilidad y productividad o hacia el cambio y mejora. El método de investigación es exploratorio con análisis documental de propuestas académicas cuestionario para definir el énfasis formativo, se aplica estadística inferencial en una muestra de pregrado y postgrado para corroborar la hipótesis. En los resultados se plantea el equilibrio formativo considerando el desarrollo de competencias ante la competencia global, y la necesidad de un proyecto académico que amplíe una visión y la proyección a partir de una misión que enfatice la formación de ingenieros industriales competitivos y de clase mundial.

Palabras clave: Enfoque de productividad, enfoque de mejora, misión, competitividad de clase mundial, ingeniero industrial.

Productivity AND IMPROVEMENT APPROACH IN INDUSTRIAL ENGINEERING OF SAN MARCOS UNIVERSITY. EXPLORATORY STUDY ORIENTED TO WORLD-CLASS COMPETITIVENESS

\section{ABSTRACT}

In order to best results in performance, industrial engineer considers the process management, human resources and seeking productivity and efficiency, also managing opportunities, the first focuses on the system in balance and operating materials, the second it is the development of tools and techniques of continuous improvement that characterizes the profession. The aim is to show the propensity of the industrial engineer to stability and productivity or to change and improvement. The method is exploratory research with academic proposals documentary analysis questionnaire to define the educational emphasis, inferential statistics is applied in a sample of undergraduate and graduate to corroborate the hypothesis. In the results the training balance considering the development of skills against global competition, and the need for an academic project to expand a vision and projecting from a mission that emphasizes the formation of industrial engineer competitive and world-class.

Keywords: Productivity approach, improvement approach, mission, world-class competitiveness, industrial engineer.

\section{Introducción}

En el contexto de la revolución industrial, donde el paradigma de la administración científica prioriza el logro de niveles óptimos de producción con miras a lograr rentabilidad máxima con adecuadas condiciones laborales, surge y se consolida la Ingeniería Industrial. El ingeniero industrial (ii) integra y unifica el sistema técnico con el sistema social para el mejor uso de recursos y empleo de la capacidad y conocimiento del personal, a fin de alcanzar el funcionamiento normal y eficiente del sistema productivo dentro de organizaciones industriales o de servicio.

A partir del segundo tercio del siglo veinte, con la llegada de la sociedad industrial y postindustrial, ha ido cambiando el enfoque de gestión, donde la renovación social y tecnológica creciente deriva en la sociedad de la información y el conocimiento, caracterizado por el acelerado ritmo de renovación tecnológica, globalización, nuevos modos de organización industrial, amplias oportunidades de negocio, conllevando al surgimiento de la nueva economía con creativos paradigmas de gestión, más contingentes y flexibles.

En este innovador contexto, el ingeniero industrial va asumiendo nuevos retos que llevan a plantear nuevas soluciones a nuevas situaciones, de manera que la profesión se enriquece continuamente con técnicas y herramientas sistemáticas y creativas para mantener el sistema en equilibrio o para cambiar el sistema productivo, en suma para resolver problemas de estabilidad y cambio en la gestión.

La pregunta de investigación del estudio es: ¿Cómo es la gestión con enfoque de estabilidad y con enfoque de cambio para mantener y mejorar los resultados de las organizaciones industriales o de servicios? La pregunta secundaria es: ¿El ingeniero industrial prefiere gestionar la estabilidad o el cambio en su tarea profesional en organizaciones?

El objetivo general del estudio es plantear el sesgo hacia el enfoque de estabilidad y el enfoque de cambio en la labor del ingeniero industrial cuando resuelve problemas de productividad y eficiencia y mejora en organizaciones. La hipótesis general es

\footnotetext{
Docente de la Facultad Ingeniería Industrial. UNMSM, Perú.

E-mail: aacevedob@unmsm.edu.pe

2 Docente de la Facultad Ingeniería Industrial. UNMSM, Perú.

E-mail: ocachayb@unmsm.edu.pe

3 Docente de la Facultad de Derecho. USMP, Perú.

E-mail: mlinaresb@unmsm.edu.pe
} 
que el enfoque de cambio en la gestión del ingeniero industrial representa un importante elemento para la eficacia de los resultados, o la misión permite elevada orientación para la gestión del cambio en la gestión de organizaciones.

\section{METODOLOGÍA DE INVESTIGACIÓN}

Esta investigación es un trabajo preliminar y exploratorio (Hernández, 2014), el diseño es de investigación empírica que realiza un estudio documental comparado de la visión, misión y perfil profesional de las escuelas que forman ii en las principales universidades del país, un estudio descriptivo acerca de las variables relevantes de formación profesional y estudio estadístico para verificar la hipótesis planteada. En la recolección de datos se ha empleado entrevista de cuestionario abierto para analizar la visión y misión de la profesión y plantear el reto de clase mundial, cuestionario cerrado con alternativas para registrar la preferencia y énfasis en la estabilidad o el cambio de los ii, trabajo de gabinete para sistematizar las respuestas mediante software informático. La recopilación de datos se ha realizado en forma aleatoria en ambiente académico de la facultad de ingeniería industrial de la UNMSM.

Para el estudio estadístico de prueba de hipótesis, la población comprende a estudiantes de pregrado y posgrado de ingeniería industrial, en una muestra se ha aplicado la herramienta de recolección de información. El tamaño de muestra se ha determinado mediante estadística inferencial para pequeñas muestras donde no se conoce la desviación estándar con un nivel de confianza de $95 \%$.

\section{MARCO CONCEPTUAL DE ESTABILIDAD Y EL CAMBIO EN ORGANIZACIONES}

\subsection{Antecedentes del estudio}

La percepción dicotómica de la realidad es planteada desde el pensamiento griego. La visión aristotélica de la realidad considera que ésta tiene una esencia inmutable, sujeta a una taxonomía y un funcionamiento estable, que no cambia; este orden solo es alterado por la percepción de los sentidos, de manera que el cambio está dentro de persona y no en la realidad circundante. Por otro lado, Heráclito añade el cambio como el continuo devenir, un flujo donde nada es permanente, todo cambia. Entre los antecedentes científicos se considera el paradigma clásico o newtoniano del mundo material, visible y concreto, estable en su composición y que se mantiene en orden y equilibrio a través de fuerzas gravitacionales y electromagnéticas, esta visión es confrontada con la visión cuántica de un mundo dinámico, imprevisible y en continuo cambio, con comportamiento inestable, probabilístico y no determinístico (Navarro y Calvo, 1990).

Inicialmente, las investigaciones académicas sobre gestión estable de las organizaciones corresponden a las teorías clásicas, la escuela de la administración científica, los neoclásicos, escuela de las ciencias de la administración, que consideran una visión equilibrada de la realidad organizacional donde se busca el óptimo funcionamiento con la mayor eficiencia y productividad (Chiavenato, 2013), el cambio se relaciona a nuevas concepciones, nuevos sistemas de creación de riqueza, donde el enfoque del cambio se torna relevante cuando enfrenta y supera a los paradigmas dominantes, el cambio refleja una nueva manera de pensar, como lo plantean Porter, Handy, Prahalad, Senge, entre otros que añaden la variable del cambio en la gestión de las organizaciones, sectores, mercados y el mundo (Gibson, 1997). Desde la teoría de sistemas se considera el enfoque holístico de "totalidad del sistema" para la resolución de los problemas (Van Gigch, 1993), planteando dos metodologías de cambio: primero, la mejora donde la organización alcanza los resultados planeados en función al diseño y especificaciones originales, corresponde al sistema en equilibrio; segundo, el rediseño y configuración del sistema a partir del cuestionamiento de su propósito en relación a sistemas mayores con los que interacciona, corresponde al sistema en cambio.

Se plantea la estabilidad y el cambio como parte de las dimensiones de gestión de la empresa (Acevedo y Linares, 2009), en el constructo del mundo de la empresa para enfrentar los problemas, la dimensión estructura se refiere a los factores ambientales estables y la dimensión del momento se refiere a las fuerzas del cambio derivado de las presiones tecnológicas y sociales, también se introducen técnicas metaheurísticas en las decisiones de gestión (Cachay, 2012) ante situaciones de inestabilidad, urgencia y cambio constante. En los estudios de Kotter,. Duck y Martin, se plantea el equilibrio en la gestión del cambio, el liderazgo en los proyectos de cambio, el cambio de mentalidad, como nuevos aspectos a considerar en las organizaciones (HBR, 2000).

En la problemática empresarial, el ingeniero industrial ha de desarrollar habilidades fundamentales para la adecuada gestión del sistema socio técnico de las organizaciones conformadas por personas, recursos y capital, buscando el equilibrio y el cambio (Tabla 1). Estas habilidades fundamentales derivan de la concepción sistémica integral de la empresa, su funcionamiento y sus resultados (Acevedo y Linares, 2012). 
Tabla 1. Habilidades del ingeniero industrial para la gestión del sistema sociotécnico.

\begin{tabular}{|c|c|c|c|c|c|c|}
\hline \multicolumn{2}{|c|}{$\begin{array}{l}\text { SISTEMA } \\
\text { SOCIOTÉCNICO }\end{array}$} & \multicolumn{2}{|c|}{ SISTEMA SOCIAL PERSONAS } & \multicolumn{2}{|c|}{ SISTEMA TÉCNICO RECURSOS } & $\begin{array}{c}\text { Paradigma } \\
\text { sociotécnico del ii }\end{array}$ \\
\hline \multicolumn{2}{|c|}{$\begin{array}{l}\text { SITUACIÓN } \\
\text { PROBLEMÁTICA }\end{array}$} & \multicolumn{2}{|c|}{$\begin{array}{c}\text { CONSENSUAR INTERESES } \\
\text { (CONFLICTO) } \\
\text { (propósito, fin, motivos, intereses) }\end{array}$} & \multicolumn{2}{|c|}{$\begin{array}{c}\text { ASIGNAR MEDIOS } \\
\text { (DILEMA) } \\
\text { (cantidad, disponibilidad, rendimiento) }\end{array}$} & $\begin{array}{l}\text { Propósito del ii } \\
\text { en la SP }\end{array}$ \\
\hline \multicolumn{2}{|c|}{ FOCO DE DECISIÓN } & $\begin{array}{l}\text { TRABAJADOR } \\
\text { PERSONA EN Sí }\end{array}$ & $\begin{array}{l}\text { PROCESO } \\
\text { TAREA EN Sí }\end{array}$ & $\begin{array}{l}\text { CAPACIDAD } \\
\text { ESPACIO }\end{array}$ & $\begin{array}{l}\text { FLUJO } \\
\text { TIEMPO }\end{array}$ & $\begin{array}{c}\text { Dimensión campo } \\
\text { de actuación }\end{array}$ \\
\hline \multicolumn{2}{|c|}{ Sistema en equilibrio } & $\begin{array}{l}\text { Disponibilidad } \\
\text { Seguridad }\end{array}$ & $\begin{array}{l}\text { Eficacia } \\
\text { Resultados } \\
\text { Eficiencia }\end{array}$ & $\begin{array}{l}\text { Balance de línea } \\
\text { Restricciones } \\
\text { Productividad }\end{array}$ & $\begin{array}{c}\text { Velocidad } \\
\text { Tiempo de ciclo } \\
\text { Funcionamiento }\end{array}$ & $\begin{array}{l}\text { Gestión de las } \\
\text { operaciones }\end{array}$ \\
\hline \multirow{2}{*}{$\begin{array}{l}\text { Sistema } \\
\text { en cambio }\end{array}$} & Mejora & $\begin{array}{l}\text { Aprendizaje } \\
\text { Liderazgo }\end{array}$ & $\begin{array}{l}\text { Capacitación } \\
\text { Motivación }\end{array}$ & Diseño de planta & $\begin{array}{l}\text { Diseño cadena de } \\
\text { suministro }\end{array}$ & $\begin{array}{l}\text { Diseño/ rediseño } \\
\text { de sistemas }\end{array}$ \\
\hline & Adaptación & $\begin{array}{l}\text { Naturaleza } \\
\text { humana }\end{array}$ & $\begin{array}{l}\text { Iso del ser } \\
\text { humano }\end{array}$ & $\begin{array}{l}\text { Técnicas ii y } \\
\text { cuantitativas }\end{array}$ & $\begin{array}{l}\text { Técnicas } \\
\text { heurísticas }\end{array}$ & $\begin{array}{l}\text { Decisiones } \\
\text { complejas }\end{array}$ \\
\hline
\end{tabular}

Fuente: Elaboración propia. Adaptado (Acevedo y Linares, 2012).

\subsection{Enfoque de estabilidad y enfoque de cambio}

Para enfrentar la problemática del mundo de las organizaciones se requiere una perspectiva sociotécnica que identifique el marco de la decisión del ii, el cual sería el enfoque de estabilidad y fun- cionamiento normal de la organización, priorizando resultados y productividad o el enfoque de cambio y reinvención mediante el diseño y rediseño del sistema empresarial (ver Figura 1).

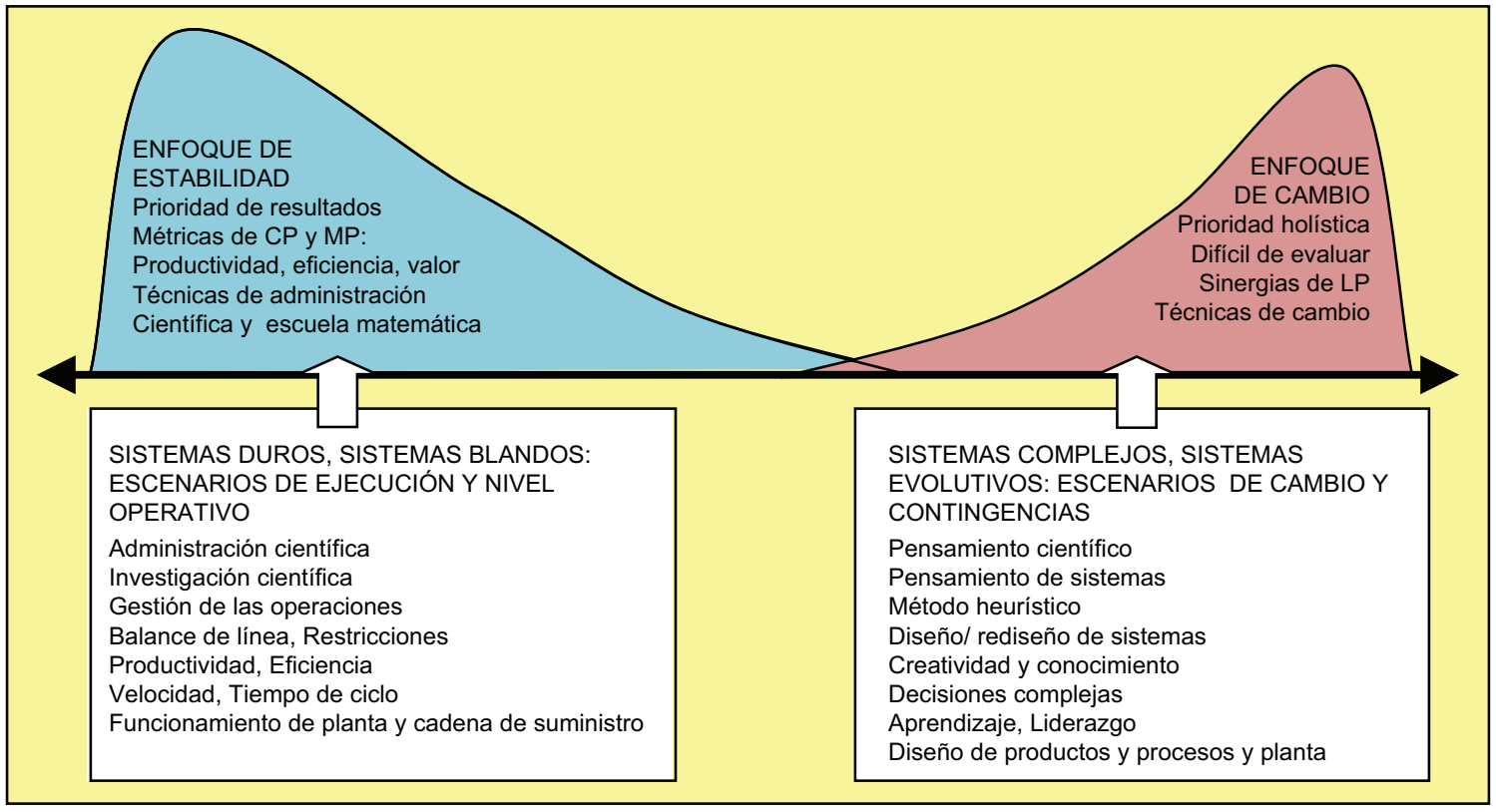

Fuente: Elaboración propia. Adaptado (Cachay, et al., 2016).

Figura 1. El enfoque de estabilidad y enfoque de cambio en las organizaciones.

En la gestión de organizaciones, el ingeniero industrial emplea el enfoque de estabilidad del proceso productivo y cadena de suministro para obtener resultados y productividad (Chase y Jacobs, 2014), es el enfoque de las empresas orientadas al negocio y la rentabilidad. Emplea el enfoque de cambio cuando se requiere rediseño drástico de la posición y futuro de organizaciones en el largo plazo, es el enfoque de estadista y visionario. Para mantener el sistema productivo en equilibrio emplea técnicas sistemáticas de la ingeniería industrial y gestión de operaciones, para generar el cambio del sistema utiliza técnicas de diseño de procesos y productos. 


\subsection{La visión y misión comparada}

La visión refleja un futuro deseado y esperado, cambiante y contingente con problemas y retos que se han de enfrentar y resolver. La misión define las dimensiones profesionales que el ingeniero industrial debe cumplir para que alcanzar el éxito y superar los retos y problemas de la gestión. En general, cada Facultad o Escuela de Ingeniería Industrial se compromete en cumplir sus propios objetivos, con mejora permanente en conocimiento, capacidades, habilidades y actitudes que permiten cubrir la misión y lograr la visión. En la Tabla 2 se muestra la visión y misión de un grupo de universidades, que compiten con el ii de UNMSM.

Tabla 2. Visión y Misión del ingeniero industrial en universidades peruanas.

\begin{tabular}{|c|c|c|c|}
\hline & UNMSM & PUCP & ULIMA \\
\hline Visión & $\begin{array}{l}\text { Ser la mejor facultad de Ingeniería Industrial } \\
\text { del sistema universitario peruano reconocida } \\
\text { internacionalmente por formar profesionales } \\
\text { líderes, con altas competencias en gestión de } \\
\text { empresas e instituciones, aplicación de tecno- } \\
\text { logía en el desarrollo sostenido del país y del } \\
\text { medio ambiente, con una formación humanista } \\
\text { integral. }\end{array}$ & $\begin{array}{l}\text { Ser una comunidad que lidere la formación de } \\
\text { profesionales competentes, comprometidos } \\
\text { y promotores del desarrollo de nuestro país. }\end{array}$ & $\begin{array}{l}\text { Universidad modelo en la formación de pro- } \\
\text { fesionales líderes, generadora de iniciativas, } \\
\text { cuyos miembros responden con celeridad y } \\
\text { eficacia a los desafíos de la sociedad. Una } \\
\text { U que evoluciona permanentemente y mantie- } \\
\text { ne el liderazgo en los campos en que desarro- } \\
\text { lla su acción. }\end{array}$ \\
\hline \multirow[t]{2}{*}{ Misión } & $\begin{array}{l}\text { Somos una facultad de la UNMSM, con alto } \\
\text { nivel académico en formación de profesionales } \\
\text { en el ámbito de la Ingeniería Industrial. Flexi- } \\
\text { bles, innovadores y capaces de responder a } \\
\text { las necesidades de la sociedad peruana, con } \\
\text { sólidos valores éticos y morales, comprometi- } \\
\text { dos con el desarrollo sostenible del país y la } \\
\text { preservación del medio ambiente. }\end{array}$ & $\begin{array}{l}\text { Formar integralmente a las personas en las } \\
\text { áreas de la ciencia, la tecnología y la gestión, } \\
\text { constituyéndonos en promotores del desa- } \\
\text { rrollo y aplicación del conocimiento y de la in- } \\
\text { vestigación que generen valor a la empresa y } \\
\text { a la sociedad, inspirados en: principios éticos, } \\
\text { excelencia profesional y calidad en nuestros } \\
\text { servicios. }\end{array}$ & $\begin{array}{l}\text { Institución académica, autónoma, sin fines de } \\
\text { lucro, integrada por profesores, alumnos y gra- } \\
\text { duados, articula docencia de alta calidad, in- } \\
\text { centiva investigación y actividades en favor de } \\
\text { la comunidad aportando profesionales idó- } \\
\text { neos con vocación de servicio, conciencia } \\
\text { ética y crítica, comprometidos con el desarrollo } \\
\text { e integración de la sociedad. }\end{array}$ \\
\hline & UNI & UNFV & URP \\
\hline Visión & $\begin{array}{l}\text { Ser líder en Latinoamérica por la excelencia } \\
\text { de sus profesionales y por su contribución al } \\
\text { desarrollo de la sociedad. }\end{array}$ & $\begin{array}{l}\text { Ser líder en el Sistema Universitario Nacio- } \\
\text { nal e Internacional, formando profesionales } \\
\text { competitivos en ingeniería, acorde con los } \\
\text { cambios científicos y tecnológicos para el de- } \\
\text { sarrollo sostenible basado en valores al servi- } \\
\text { cio de la sociedad. }\end{array}$ & $\begin{array}{l}\text { Alcanzar y sostener el liderazgo en la for- } \\
\text { mación de ingenieros industriales prepara- } \\
\text { dos para desempeñarse con éxito en empre- } \\
\text { sas e instituciones, en el país o el extranjero, } \\
\text { enfrentar retos, lograr mejores resultados en } \\
\text { calidad y productividad. }\end{array}$ \\
\hline Misión & $\begin{array}{l}\text { Generar conocimiento y formar profesionales } \\
\text { con capacidad de diseño, investigación, in- } \\
\text { novación y enfoque sistémico para solucio- } \\
\text { nar necesidades de la sociedad; contando con } \\
\text { personal docente y administrativo calificado, } \\
\text { comprometido y motivado e infraestructura } \\
\text { tecnológica actualizada, conformando una co- } \\
\text { munidad académica, científica, tecnológica y } \\
\text { humanista }\end{array}$ & $\begin{array}{l}\text { Somos una Facultad Académica comprome- } \\
\text { tida con la formación integral de ingenieros } \\
\text { industriales, de sistemas, agroindustriales y de } \\
\text { transportes, con base científica, tecnológica } \\
\text { y humanista al servicio del desarrollo de la } \\
\text { sociedad. }\end{array}$ & $\begin{array}{l}\text { Formar Ingenieros Industriales altamente com- } \\
\text { petentes, preparados para desempeñarse con } \\
\text { eficiencia, eficacia y profesionalismo con } \\
\text { conocimiento de ciencias básicas e ingeniería, } \\
\text { formación humanística, para adaptarse a las } \\
\text { tendencias de la ciencia y tecnología, actuan- } \\
\text { do con responsabilidad ética y social }\end{array}$ \\
\hline \multicolumn{4}{|c|}{$\begin{array}{l}\text { Fuente: Elaboración propia. UNMSM: Universidad Nacional Mayor de San Marcos (UNMSM-FII, 2015), PUCP: Pontificia Universidad Católica del Perú } \\
\text { (PUCP-FI, 2015), ULIMA: Universidad de Lima (UL-FI, 2015), UNI: Universidad Nacional de Ingeniería (UNI-FII, 2015), UNFV: Universidad Nacional Fe- } \\
\text { derico Villarreal (UNFV-FIIS, 2015), URP: Universidad Ricardo Palma (URP-FII, 2015). }\end{array}$} \\
\hline
\end{tabular}

Fuente: Elaboración propia. Adaptado (Cachay, et al., 2016).

Respecto a la visión, las facultades plantean un liderazgo semejante, formulando un liderazgo profesional. UNMSM propone ser la mejor facultad formando profesionales líderes competentes en gestión, PUCP plantea liderar la formación de profesionales competentes comprometidos con el desarrollo, ULIMA y URP enfatizan profesionales líderes que responden con celeridad y eficacia, UNI y UNFV plantean liderazgo internacional.

Las diferencias se perciben en la misión. La UNMSM define un profesional flexible e innovador con equilibrio entre la parte de gestión y la parte de innovación y cambio, la PUCP forma promotores del desarrollo, la ULIMA forma profesionales con vocación de servicio, la UNI enfatiza el cambio y el enfoque sistémico, la UNFV presenta una formación integral con base científica, tecnológica y humanista, la URP enfatiza la eficiencia, eficacia y profesionalismo. Solamente PUCP, ULIMA y UNI consideran investigación en su misión.

Un aspecto básico de la formación profesional es el propósito explícito de la universidad que se orienta hacia fines de la sociedad y sectores, o de intereses de grupo. Por un lado, UNMSM busca competitividad y satisfacción del consumidor o usuario, ULIMA busca el desarrollo sostenible de la sociedad, UNI se orienta al conocimiento de la realidad y de los recursos del país, UNFV y URP coinciden en el de- 
sarrollo de la pequeña empresa. La U PUCP afirma que la finalidad del profesional ii, es convertirse en gerente y director de personal.

\subsection{La Misión en acción}

Principios en acción. En el propósito de referente académico nacional e internacional, la FII-UNMSM define que el papel de las autoridades y docentes es guiar y otorgar estructura a los cambios en base a su conocimiento y experiencia; el papel de emprendimiento y espíritu innovador frente a los retos y el cambio corresponden a los jóvenes estudiantes.

Las acciones estratégicas de la FII. La Figura 2 presenta las acciones que la FII desarrolla para alcanzar el propósito de ser referente académico y de investigación. El esquema elaborado es referencial, en el sentido que una misma estrategia tiene objetivos de estabilidad consolidando capacidades, y objetivos de cambio que visionan en futuro y la transformación a través de la propia reinvención (Cachay, 2015).

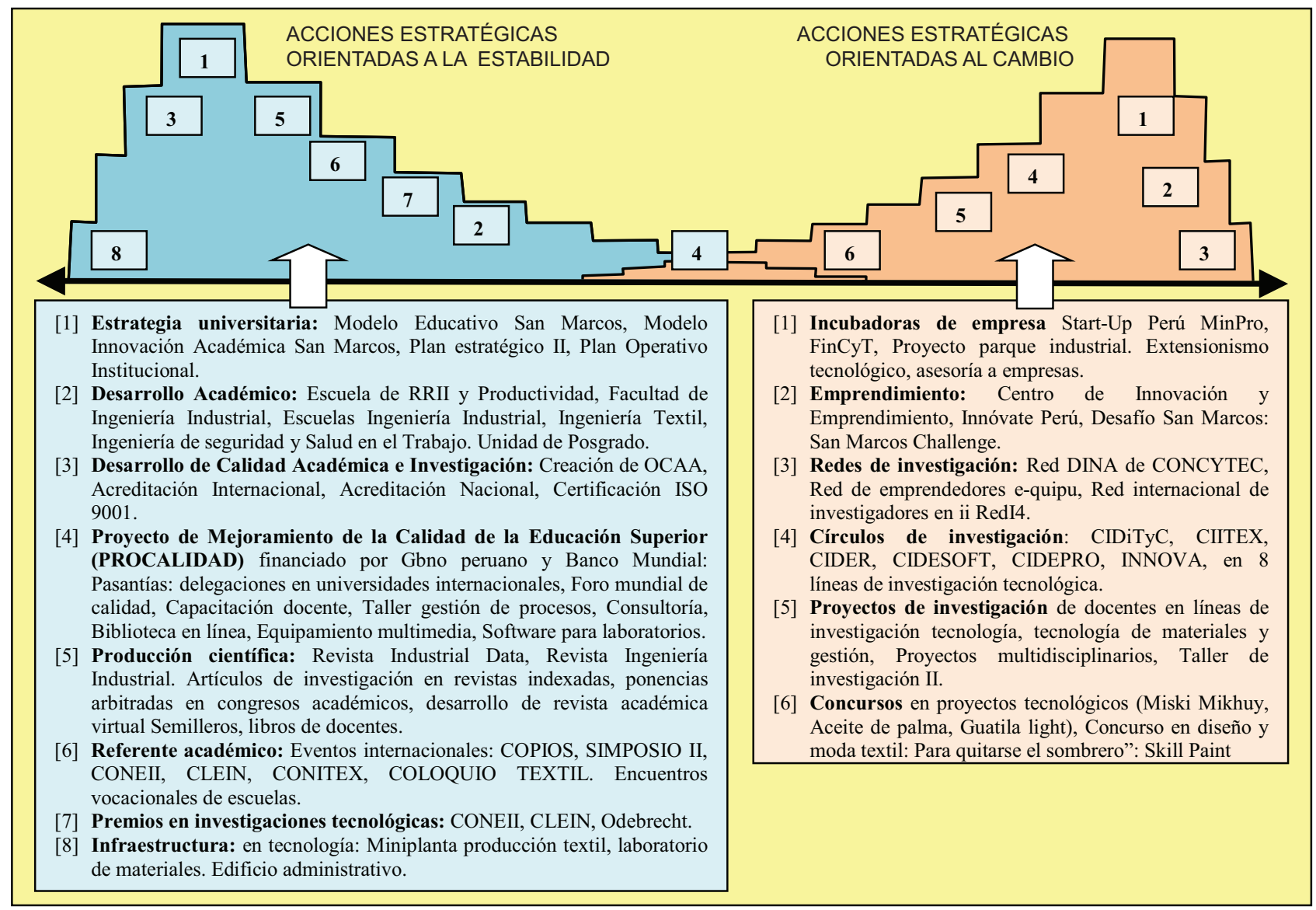

Fuente: Elaboración propia

Figura 2. Acciones estratégicas orientadas a la estabilidad y el cambio.

\subsection{Brechas en el perfil profesional}

\section{Orientación del currículo}

De acuerdo al modelo conceptual de las 4 dimensiones MC4D (Acevedo, 2010), los planes curricu- lares de ingeniería industrial se agrupan según los niveles de resolución de problemas. Comparativamente, en la Tabla 3 se muestran los porcentajes de cursos en cada nivel. 
Tabla 3. Porcentaje de cursos por niveles de resolución. Universidades seleccionadas.

\begin{tabular}{|c|c|c|c|c|c|c|}
\hline \multirow{2}{*}{ Nivel de resolución } & \multicolumn{5}{|c|}{$\%$ de cursos en cada nivel de resolución en porcentaje } & \multirow{2}{*}{$\begin{array}{c}\% \text { Promedio } \\
\text { Total }\end{array}$} \\
\hline & UNMSM & PUCP & ULIMA & UNI & UNFV & \\
\hline NE Nivel estratégico-directivo & 14 & 19 & 15 & 10 & 10 & 14 \\
\hline NG Nivel táctico gerencial & 16 & 22 & 27 & 27 & 24 & 23 \\
\hline OPE Nivel táctico operativo & 24 & 20 & 21 & 20 & 24 & 22 \\
\hline TEC Nivel básico técnico & 24 & 17 & 14 & 11 & 19 & 17 \\
\hline BAS Nivel básico ciencias-humanidades & 22 & 21 & 23 & 32 & 24 & 25 \\
\hline TOTAL & 100 & 100 & 100 & 100 & 100 & 100 \\
\hline
\end{tabular}

Fuente: Elaboración propia. Adaptado de (Vega et al., 2015).

Las universidades presentan diferente sesgo. La PUCP presenta mayor perfil estratégico (NE), la ULIMA muestra mayor orientación hacia el nivel gerencial y la UNI hacia el nivel de formación básica. En Tabla 4 se observa que PUCP y ULIMA son semejantes ya que ambos se dirigen a la formación académica a nivel estratégico, UNMSM, UNFV y UNI se orientan a la formación técnica y básica, aunque UNI presenta orientación hacia niveles NE+NG.

Tabla 4. Porcentaje de cursos por niveles de resolución agregados. Universidades seleccionadas.

\begin{tabular}{|c|c|c|c|c|c|c|}
\hline \multirow{2}{*}{ Nivel de resolución } & \multicolumn{5}{|c|}{$\%$ de cursos en cada nivel de resolución en niveles agregados } & \multirow{2}{*}{$\begin{array}{c}\% \% \\
\text { Promedio } \\
\text { Total }\end{array}$} \\
\hline & UNMSM & PUCP & ULIMA & UNI & UNFV & \\
\hline $\mathrm{NE}+\mathrm{NG}$ & 30 & 41 & 42 & 37 & 33 & 37 \\
\hline OPE & 24 & 20 & 21 & 20 & 24 & 22 \\
\hline TEC+BAS & 46 & 39 & 37 & 43 & 43 & 42 \\
\hline TOTAL & 100 & 100 & 100 & 100 & 100 & 100 \\
\hline
\end{tabular}

Fuente: Elaboración propia.

La Figura 3 señala que la UNMSM presenta la mayor proporción de cursos técnicos y básicos y la menor proporción de cursos de nivel estratégico-gerencial, lo que indica que la formación académica de la UNMSM enfatiza la formación para la operación y planta, al contrario de la ULIMA que enfatiza la formación gerencial y estratégica. Una generalización preliminar señalaría que el enfoque de estabilidad se ubica en los cursos operativos mientras que el enfoque de cambio se ubica en los cursos de estrategia y gerencia.

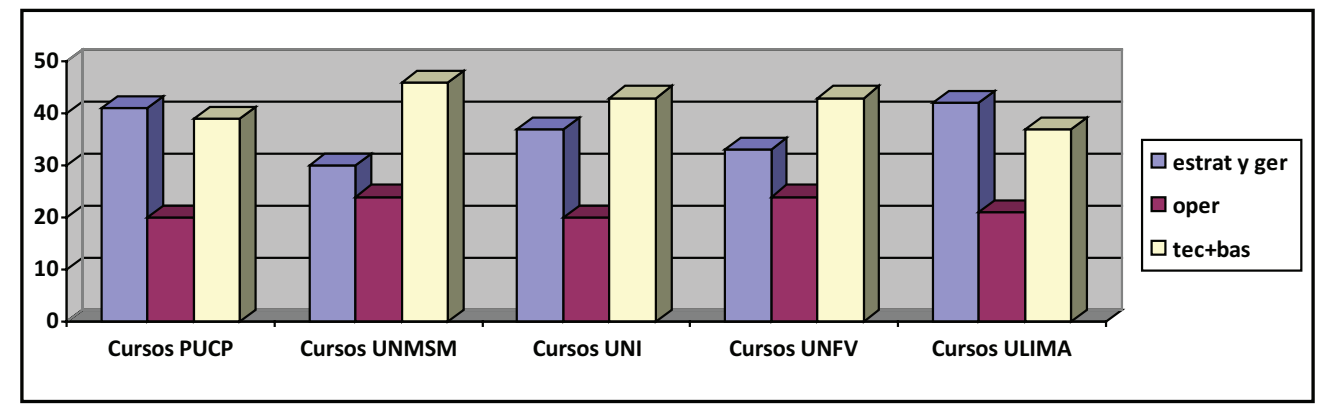

Fuente: Elaboración propia.

Figura 3. Orientación general de los cursos, por universidades. 


\section{La brecha percibida}

Según el perfil, el ingeniero industrial de la UNMSM es un profesional comprometido que genera bienestar compartido, integrando formación humanística, científica y tecnológica para cubrir el desarrollo humano y las necesidades del mercado. La percep- ción de los estudiantes sobre este perfil se presenta en la Figura 4. La menor brecha se encuentra en los temas de resultados tangibles y empleo de técnicas sistemáticas y la mayor brecha se ubica en los temas de investigación y cambio, constituyendo una posible debilidad formativa.

\begin{tabular}{|l|l|l|l|l|l|l|l|l|l|l||}
\hline Actitud del ingeniero industrial & 1 & 2 & 3 & 4 & 5 & 6 & 7 & 8 & 9 & 10 \\
\hline $\begin{array}{l}\text { Emprendimiento y mejora de la } \\
\text { calidad de vida en la sociedad }\end{array}$ & & & & & & & & & & \\
\hline $\begin{array}{l}\text { Optimización de procesos, } \\
\text { productos y servicios }\end{array}$ & & & & & & & & & & \\
\hline $\begin{array}{l}\text { Énfasis en formación científica y } \\
\text { tecnológica }\end{array}$ & & & & & & & & & & \\
\hline $\begin{array}{l}\text { Énfasis en el empleo de técnicas } \\
\text { matemáticas y cuantitativas }\end{array}$ & & & & & & & & & & \\
\hline $\begin{array}{l}\text { Interés por la investigación y } \\
\text { creación de conocimiento }\end{array}$ & & & & & & & & & & \\
\hline $\begin{array}{l}\text { Interés por la investigación en } \\
\text { ciencias de materiales }\end{array}$ & & & & & & & & & & \\
\hline $\begin{array}{l}\text { Interés en iniciar el cambio y } \\
\text { adaptación a los cambios }\end{array}$ \\
\hline
\end{tabular}

Fuente: Elaboración propia.

Figura 4. Perfil percibido de actitud hacia estabilidad y cambio en la UNMSM.

\section{RESULTADOS DEL ESTUDIO}

A partir de la prueba de hipótesis, donde la hipótesis nula y la hipótesis alternativa, son: $\mathbf{H}_{0}$ : El enfoque de cambio del ii no representa un aporte importante para los resultados en la gestión de organizaciones. $\mathbf{H}_{1}$ : El enfoque de cambio del ii representa un aporte importante para los resultados en la gestión de organizaciones.
De manera que: $\mathrm{H}_{0}: \mu_{1}=\mu_{2} ; \mathrm{H}_{1}: \mu_{1}<\mu_{2}$. Donde: $\mu_{1}$ : Media de preferencia de enfoque de cambio y mejora; $\mu_{2}$ : Media de preferencia de enfoque de estabilidad y resultados.

Para un nivel de significación $\alpha=0.05$ y nivel de confianza $1-\alpha=0.95$, se han calculado los estadísticos y la prueba de hipótesis que se muestra en la Tabla 5.

Tabla 5. Prueba de hipótesis. Prueba de muestras relacionadas.

\begin{tabular}{|c|c|c|c|c|c|c|c|c|c|}
\hline & & \multicolumn{5}{|c|}{ Diferencias relacionadas } & \multirow{3}{*}{$\mathbf{t}$} & \multirow{3}{*}{ gl } & \multirow{3}{*}{$\begin{array}{c}\text { Sig. } \\
\text { (bilateral) }\end{array}$} \\
\hline & & \multirow{2}{*}{ Media } & \multirow{2}{*}{$\begin{array}{l}\text { Desviación } \\
\text { típ. }\end{array}$} & \multirow{2}{*}{$\begin{array}{l}\text { Error típ. } \\
\text { de la media }\end{array}$} & \multicolumn{2}{|c|}{$\begin{array}{l}\text { 95\% Intervalo de confianza } \\
\text { para la diferencia }\end{array}$} & & & \\
\hline & & & & & Inferior & Superior & & & \\
\hline Par 1 & $\begin{array}{l}\text { CAMBIO - } \\
\text { ESTABILIDAD }\end{array}$ & $-2,760$ & 5,410 & 1,082 & $-4,993$ &,- 527 & $-2,551$ & 24 & ,018 \\
\hline
\end{tabular}

Fuente: Elaboración propia con SPSS. 
Área bajo la curva normal para nivel de confian-

za de 0.95. Se muestra en la Figura 5.

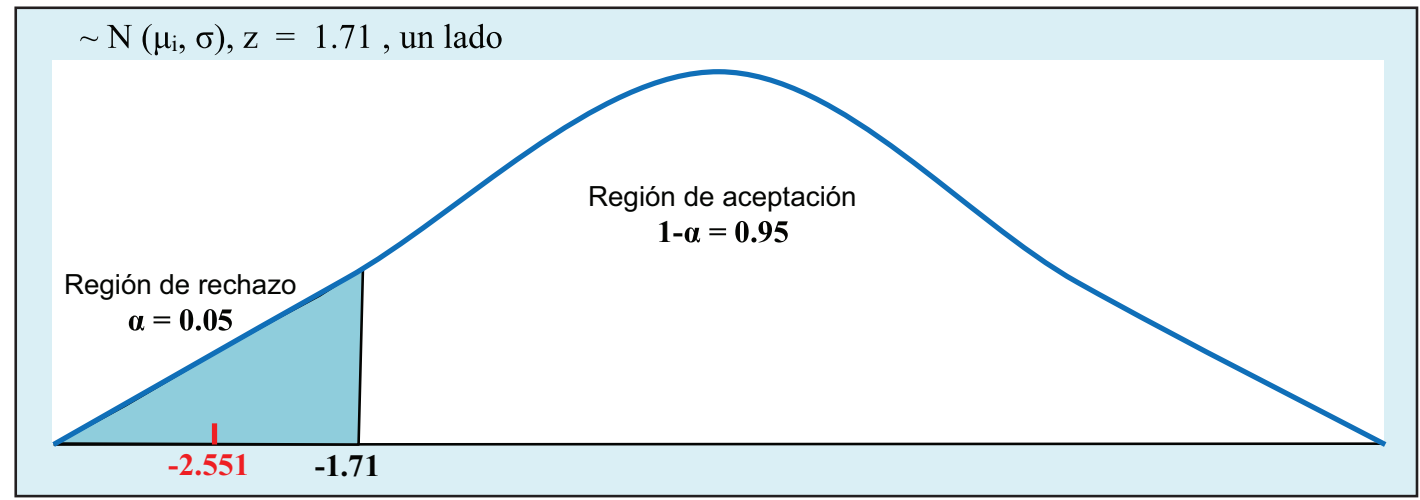

Fuente: Elaboración propia con SPSS.

Figura 5. Corroboración de hipótesis - Enfoque estabilidad y enfoque de cambio.

Los resultados indican que, según la prueba de hipótesis:

- Se rechaza la hipótesis nula a un nivel de significación de 0.05 .

- No se rechaza la hipótesis alternativa un nivel de significación de 0.05 .

No es posible aceptar la hipótesis $\mathrm{H}_{0}$ que afirma que los ingenieros industriales no presentan orientación definida hacia enfoque de estabilidad o enfoque de cambio. Alternativamente no es posible rechazar la hipótesis alternativa que afirma que los ingenieros industriales presentan orientación mayor hacia el enfoque de estabilidad. Por lo tanto, según los resultados de la validación estadística de hipótesis, se corrobora que, en la muestra de ingenieros industriales, se emplea mayormente el criterio de estabilidad en la resolución de problemas.

\section{DISCUSIÓN DE LOS RESULTADOS}

Se ha rechazado la hipótesis nula ya que no ha sido posible corroborar que no existe sesgo del ingeniero industrial hacia el enfoque de estabilidad por encima del enfoque de cambio, de manera que la misión y la formación profesional plantean una preferencia hacia el empleo de criterios y técnicas enfocadas en el funcionamiento ordenado del sistema organizativo. Estos resultados indican que la visión y la misión plantean mayor prioridad hacia los criterios de estabilidad con énfasis en productividad y eficiencia, mientras que los criterios de cambio tiene menor prioridad, dentro del proceso formativo de manera que se podrían configurar, en el futuro, debilidades competitivas ante las presiones del entorno profesional cambiante.
El estudio empírico se presenta en la Figura 6, la orientación en los criterios de elección de los ingenieros industriales se dirige hacia la estabilidad $(72 \%)$ y el buen funcionamiento, lo que indica énfasis en los resultados de corto plazo, en menor medida emplea técnicas de cambio cualitativo. El propósito de cambio es menor (28\%), lo que indica que el énfasis pragmático de la profesión facilita la estabilidad en las organizaciones y un reducido énfasis en herramientas innovadoras y creativas. Se resalta las diferencias en el tratamiento (o prioridad) en el sesgo a la estabilidad por sobre el cambio del ii cuando resuelve problemas.

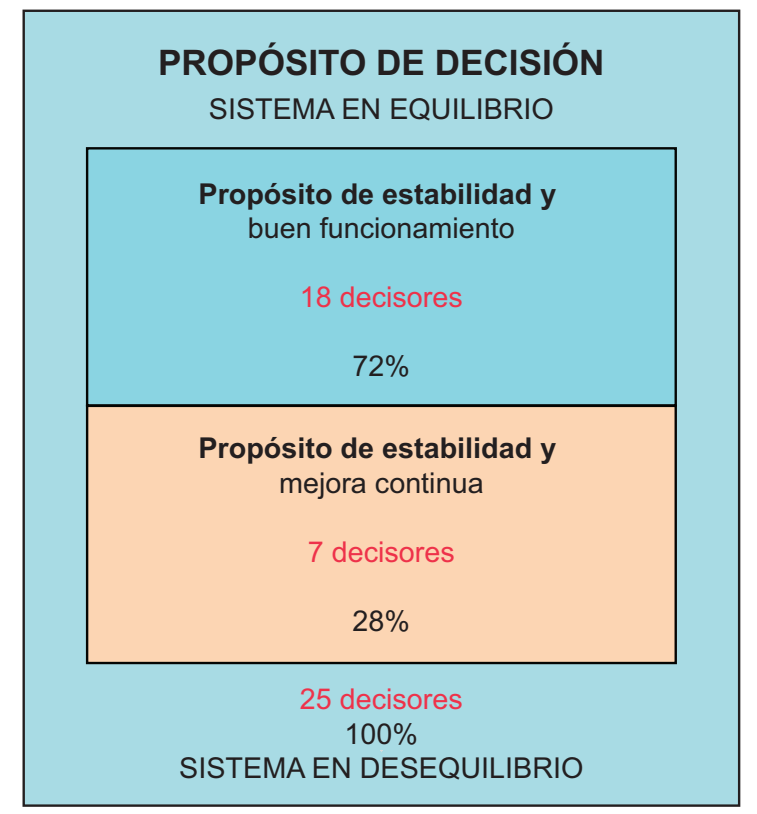

Fuente: Elaboración propia.

Figura 6. Preferencia por estabilidad o cambio en el ii. 
De esta manera se define el aporte social del estudio. El planteo de toma de decisiones con criterios preferentes de estabilidad define la fortaleza competitiva del ingeniero industrial, en la tarea de gestión de organizaciones. En la Figura 7 se muestra la
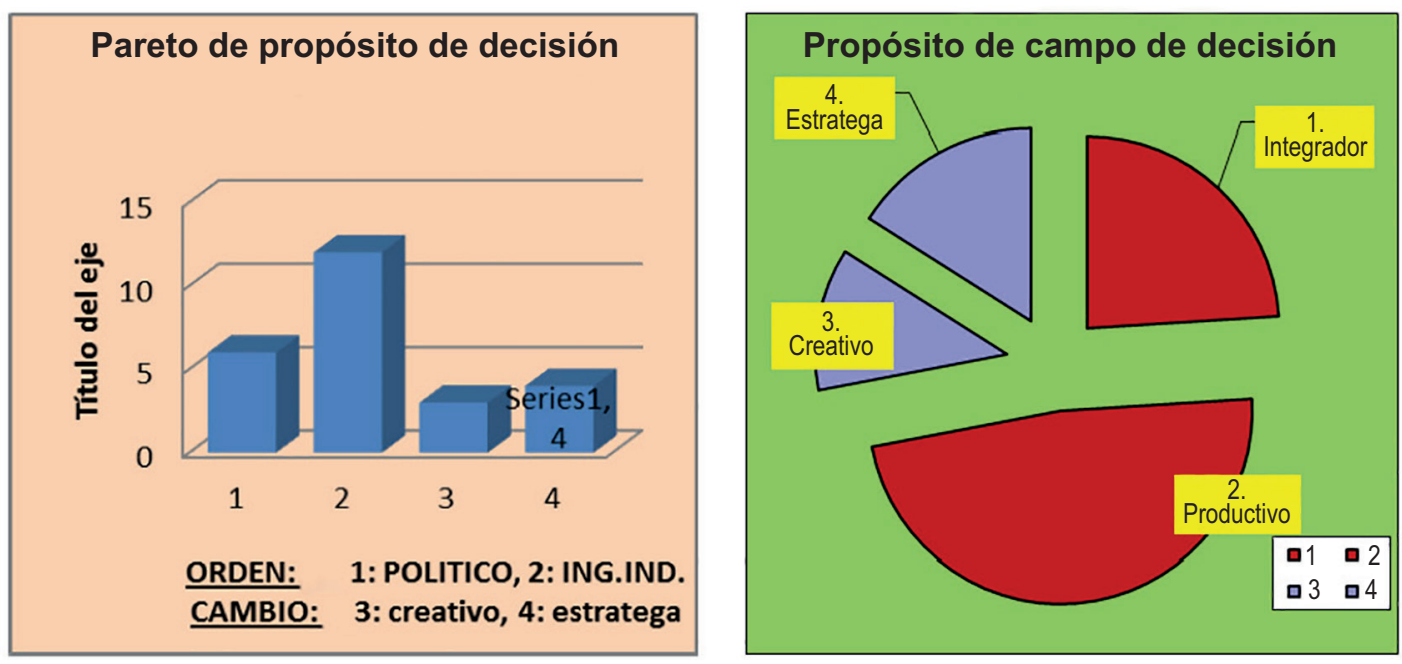

Fuente: Elaboración propia. prioridad productiva (resultados de los procesos) e integradora (uso de recursos), otros aspectos como estrategia y creatividad cercanos a la predisposición al cambio, se percibe como poco prioritarios.

Figura 7. Perfil de la preferencia del ii y su fortaleza decisional.

El estudio pretende contribuir al desarrollo de nuevas perspectivas en la tarea profesional del ingeniero industrial, donde el enfoque clásico en gestión de la productividad y operación estable de procesos y organizaciones, se ha de enriquecer con la gestión del cambio planteando la adopción de criterios que faciliten el posterior desarrollo de herramientas y técnicas para el cambio e innovación en las organizaciones. Estos nuevos métodos han de considerar la capacidad de entender el contexto, predecir situaciones y prever los posibles efectos. Complementariamente se intenta la formulación de criterios que relacionen e integren la capacidad de gestionar el entorno estable con la posibilidad de prever el entorno cambiante donde se enmarca la acción de las organizaciones, constituyendo el campo ampliado de las decisiones de resolución de problemas del ii.

\section{CONCLUSIONES}

El estudio permite la obtención de nuevos planteamientos para la gestión de las empresas y para la gestión de la innovación.

El perfil profesional del ingeniero industrial de la UNMSM se sustenta en fortalezas en gestión de la organización en funcionamiento estable, que conlleva resultados adecuados en el medio y corto plazo. La formación profesional enfatiza la gestión de resultados, la productividad y la eficiencia. Se ha identificado como fortaleza mayor, el énfasis en la estabilidad, la debilidad que se deben reforzar es la formación en gestión del cambio.

Los perfiles del currículo de cursos de los ingenieros industriales presentan sutiles semejanzas entre las universidades nacionales, enfocadas en cursos básicos, frente las universidades privadas enfocadas en cursos de gestión.

El aporte general del estudio es la contribución al estudio de nuevos enfoques de la ingeniería industrial, desde la gestión de productividad y operación estable de los procesos y organizaciones, hacia la gestión del cambio adoptando nuevos métodos y herramientas con criterios de cambio e innovación en las organizaciones.

La percepción de oportunidades globales ha de permitir que el profesional de ingeniería industrial de la UNMSM cumpla su misión superior de creación y consolidación de empresas, gestión de organizaciones pequeñas, medianas o grandes, aporte al conocimiento aplicado y tecnológico para el desarrollo y finalmente, aportar al bienestar social. 


\section{RECOMENDACIONES}

La percepción de oportunidades globales ha de permitir que el profesional de ingeniería industrial de la UNMSM cumpla su misión superior de creación y consolidación de empresas, gestión de organizaciones pequeñas, medianas o grandes, aporte al conocimiento aplicado y tecnológico para el desarrollo y finalmente, aportar al bienestar social.

Los ingenieros industriales han de considerar las tendencias y oportunidades que surgen en el ambiente mundial de manera que desarrolle plenamente su potencialidad de gestionar organizaciones en diferentes contextos, dentro de la tendencia de consolidar una formación profesional de clase mundial para afrontar el reto de la globalización y competencia mundial.

Se plantea ampliar el estudio para desarrollar los elementos que permitan crear y consolidar una formación académica de excelencia de manera que se brinda a la sociedad un ingeniero industrial competitivo de clase mundial.

\section{REFERENCIAS BIBLIOGRÁFICAS}

[1] Acevedo, A. y Linares C. (2012). El enfoque y rol del ingeniero industrial para la gestión y decisión en el mundo de las organizaciones. Industrial Data, 15(1), 9-24, http://dx.doi. org/10.15381/idata.v15i1.6236

[2] Acevedo, A. (2010). El modelo conceptual de las 4 Dimensiones para la resolución de problemas. Industrial Data, 13(2), 15-24, http:// dx.doi.org/10.15381/idata.v13i2.6179

[3] Acevedo, A., y Linares, C. (2009). La resolución de problemas en el mundo de la empresa. Estudio exploratorio sobre relativismo decisional. Industrial Data, 12(2), 81-88, http://dx.doi. org/10.15381/idata.v12i2.6136

[4] Cachay, O. (2015). 50 años reinventándonos. Revista Ingeniería Industrial 5 (7), 1.

[5] Cachay, O. (2012). Modelo metaheurístico aplicado al problema de enrutamiento de vehículos cisterna en la cadena de suministro de combustible en la ciudad de Lima. USA: Editorial Académica Española.

[6] Cachay, O., Linares, C., Acevedo, A., Ponce, W. (2016). Enfoque de estabilidad y enfoque de cambio en la perspectiva del ingeniero industrial. Estudio exploratorio sobre propensión decisional. Memorias IX Simposio Internacio- nal de Ingeniería Industrial: Actualidad y Nuevas Tendencias 2016. Universidad Federal Rio Grande Do Sul. Porto Alegre, Brasil.

[7] Chase, R. y Jacobs, F. (2014). Administración de Operaciones. Producción y cadena de suministro. 13ra. edición. México. Mc Graw Hill.

[8] Chiavenato, I. (2013). Introducción a la teoría general de la administración. 8va. Edición. México. McGraw Hill.

[9] Gibson, R. (1997). Repensando el futuro. Colombia.Grupo Editorial Norma.

[10] HBR Harvard Business Review (2000). Gestión del cambio. Colombia.Ediciones Deusto.

[11] Hernández, R., Fernández, C., Baptista, P. (2014). Metodología de la investigación. $6^{a}$. edición. México. Mcgraw Hill.

[12] Navarro y Calvo (1990). Historia de la Filosofía. España. Grupo Anaya S.A.

[13] PUCP-FI. Facultad de Ingeniería (2015). Recuperado de: http://facultad.pucp.edu.pe/ingenieria/facultad/mision-y-vision/

[14] UL-FI. Universidad de Lima (2015). Recuperado de: http://www.ulima.edu.pe/acerca-de/ mision-vision-y-valores

[15] UNFV-FIIS (2015). Recuperado de: http:// www.unfv.edu.pe/facultades/Fiis/nuestra-facultad/mision-y-vision

[16] UNI-FII. Universidad Nacional de Ingeniería. Facultad de Ingeniería industrial (2015). Recuperado de http://www.fiis.uni.edu.pe/facultad

[17] UNMSM-FII (2015). Recuperado de http://industrial.unmsm.edu.pe/

[18] UNMSM-FII (2015). El industrialito. Guía Estudiantil de la Facultad de Ingeniería Industrial. Lima-Perú.

[19] URP-FII (2015). Recuperado de http://www. urp.edu.pe/ingenieria.industrial/portal/index. php?urp=vision

[20] Van Gigch, J. (1993). Teoría general de sistemas. 3ra. reimpresión. México. Ed. TRILLAS.

[21] Vega, L., Cachay, O., Acevedo, A. (2015). El sesgo profesional en la enseñanza de la Ingeniería industrial. Estudio preliminar en el ambiente académico peruano. Revista Semilleros. 4(1). Recuperado de: https: //www. dropbox.com $/ \mathrm{sh} / 5$ t0beq $1 \mathrm{k} 3 \mathrm{mc} 7 \mathrm{klj} / \mathrm{AACBWE}$ Ya1UkmLrwZvispvogga?dl=0 